\title{
Low birthweight and premature birth are both associated with type 2 diabetes in a random sample of middle-aged Danes
}

\author{
K. Pilgaard • K. Færch • B. Carstensen • P. Poulsen • \\ C. Pisinger • O. Pedersen • D. R. Witte $\cdot$ T. Hansen • \\ T. Jørgensen • A. Vaag
}

Received: 13 August 2010/Accepted: 23 August 2010/Published online: 22 September 2010

(C) Springer-Verlag 2010

\begin{abstract}
Aims/hypothesis We studied the associations of size at birth and prematurity with type 2 diabetes, insulin sensitivity and beta cell function in the Danish population-based Inter99 study (ClinicalTrials.gov NCT00289237).

Methods Information about size at birth and prematurity was identified from original midwife records in 4,744 middle-aged Danes. Type 2 diabetes status, insulin sensitivity
\end{abstract}

K. Pilgaard $(\bowtie) \cdot$ K. Færch $\cdot$ B. Carstensen $\cdot$ D. R. Witte $\cdot$

A. Vaag

Steno Diabetes Center,

Niels Steensens Vej 1,

2820 Gentofte, Denmark

e-mail: kapg@steno.dk

C. Pisinger · T. Jørgensen

Research Center for Prevention and Health, Glostrup Hospital,

Glostrup, Denmark

P. Poulsen

Novo Nordisk,

Bagsværd, Denmark

O. Pedersen $\cdot$ T. Hansen

Hagedorn Research Institute,

Gentofte, Denmark

T. Hansen

Faculty of Health Sciences, University of Southern Denmark, Odense, Denmark

O. Pedersen · T. Jørgensen

Faculty of Health Sciences, University of Copenhagen,

Copenhagen, Denmark

O. Pedersen

Faculty of Health Sciences, University of Aarhus,

Aarhus, Denmark
(Matsuda index) and beta cell function (disposition index) were assessed using a $75 \mathrm{~g}$ oral glucose tolerance test. Participants born prematurely were compared with a group of at-term participants born small for gestational age.

Results An increase in birthweight of $1 \mathrm{~kg}$ was associated with a $51 \%$ (OR $0.49,95 \%$ CI $0.35-0.69)$ reduced risk of type 2 diabetes. Ponderal index, reflecting thinness at birth, was associated with type 2 diabetes to the same extent as birthweight. The prevalence of type 2 diabetes was increased to a similar degree in participants born prematurely and participants born small for gestational age, although the former had a higher ponderal index at birth. In addition, birthweight $z$-scores, reflecting fetal growth rate, were unrelated to the risk of type 2 diabetes and to other measures of glucose regulation in participants born prematurely. While low birthweight was inversely associated with insulin sensitivity and beta cell function, prematurity was associated solely with decreased insulin sensitivity.

Conclusions/interpretation While the association between birthweight and risk of type 2 diabetes is mediated via combined effects on beta cell function and insulin sensitivity, prematurity seems to influence risk of type 2 diabetes via attenuated insulin sensitivity only and independently of fetal growth rates.

Keywords Beta cell function - Birthweight - Insulin sensitivity Premature Type 2 diabetes

\section{Abbreviation \\ SGA Small for gestational age}




\section{Introduction}

Low birthweight has previously been associated with type 2 diabetes $[1,2]$. However, only few studies have examined this association in a random sample of the population and used an OGTT to diagnose type 2 diabetes. Reduced insulin sensitivity has been reported to be a mediator between low birthweight and type 2 diabetes [2, 3], while beta cell dysfunction has been associated with low birthweight in some [1] but not all studies [2].

Beside low birthweight, prematurity as such has been associated with reduced insulin sensitivity in young patients [4]. A recent Swedish register-based study reported that prematurity was associated with an increased risk of type 2 diabetes independently of birthweight [5].

We aimed to examine the associations between both size at birth and prematurity, and the risk of type 2 diabetes in a Danish study sample. We also examined the extent to which birthweight and/or prematurity are associated with insulin sensitivity and/or beta cell function.

\section{Methods}

Study background The Inter99 study is a Danish intervention study aimed at reducing the incidence of ischaemic heart disease and type 2 diabetes [6] (www.Inter99.dk, accessed 7 September 2010). From a population-based sample $(n=61,301)$ of 30 - to 60 -year-old individuals, a random sample of 13,016 was invited. The participation rate was $52.5 \%(n=6,784)$. We used data from the baseline examinations, which were performed from 1999 to 2001. Participants gave written informed consent prior to inclusion. The protocol was in accordance with the Helsinki Declaration, approved by the local ethics committee (KA98155) and registered with ClinicalTrials.gov (NCT00289237).

Participants completed a self-administered questionnaire on health, socioeconomic factors, lifestyle and maternal/paternal diabetes status (yes/no/unknown). Lifestyle variables included physical activity, diet, alcohol consumption and smoking. After an overnight fast, a $75 \mathrm{~g}$ OGTT was performed. Venous samples for measurement of plasma glucose and serum insulin concentrations were taken at 0,30 and $120 \mathrm{~min}$ and analysed as previously described [6]. Type 2 diabetes was diagnosed according to the WHO 1999 criteria [7].

Midwife records of 4,744 participants were identified as previously described [8]. Ponderal index, i.e. birthweight $(\mathrm{kg}) /$ length $\left(\mathrm{m}^{3}\right)$, was calculated as a measure of infant body composition. We included 4,644 participants born as singletons and without type 1 diabetes in this study.

Prematurity Participants born prematurely (before week 37) were compared with a group of singletons born at term (weeks 37-42) and matched for birthweight; this group was referred to as small for gestational age (SGA). The remaining participants born at term were considered appropriate for gestational age. Detailed information on gestational age was available in 363 of the 443 participants born prematurely and fetal growth rates were assessed using birthweight $z$-scores, calculated as: (birthweight-normal birthweight for gestational age)/SD of normal birthweight. A Swedish reference curve for normal weight was used [9].

Insulin sensitivity and beta-cell function Insulin sensitivity was assessed by HOMA of insulin sensitivity (1/HOMA of insulin resistance [10]) and a modified Matsuda index [11]. Areas under the curve for plasma glucose and serum insulin were calculated using a trapezoidal method. Beta cell function (disposition index) was assessed as the product of insulin secretion $\left(\mathrm{AUC}_{\text {insulin }} / \mathrm{AUC}_{\text {glucose }}\right)$ and insulin sensitivity (modified Matsuda index). Participants with known type 2 diabetes were excluded from the analysis of insulin sensitivity, insulin secretion and beta cell function.

Statistical analysis The association between birthweight and measures of glucose regulation and type 2 diabetes was investigated in four steps. Model 1 adjusted for age and sex. Model 2 additionally adjusted for adult BMI. Model 3 additionally adjusted for maternal parity and parental diabetes status, with missing or 'unknown' information on parental diabetes treated as 'no diabetes'. Model 4 further adjusted for socioeconomic factors and lifestyle variables (physical activity, diet, alcohol consumption and smoking).

Risk of type 2 diabetes was assessed by logistic regression analysis. Multivariate linear regression analyses were performed to study the relationship between birthweight or prematurity and OGTT-derived measures of glucose regulation. The outcome variables presented in Table 1 were $\log _{\mathrm{e}}$-transformed to meet the assumption of normality. Results are in percentage change per $1,000 \mathrm{~g}$ increase in birthweight. We did not use correction for multiple testing, as our work was based on the predefined rationale of a potential association between low birthweight and prematurity, and (1) type 2 diabetes, (2) reduced insulin sensitivity and (3) beta cell dysfunction as previously described. SAS version 9.1 (SAS Institute, Cary, NC, USA) was used for statistical analysis.

\section{Results}

In this random sample of Danes, $69 \%(n=154)$ of diabetes cases were screen-detected; the prevalence of type 2 diabetes was $6.5 \%$ for men and $3.8 \%$ for women.

An increase in birthweight of $1 \mathrm{~kg}$ was associated with a $51 \%$ (OR 0.49; 95\% CI 0.35-0.69; $p<0.001$ ) lower risk of 
Table 1 Risk of type 2 diabetes and percentage differences in OGTT-derived measures of glucose regulation associated with a 1,000 $\mathrm{g}$ increase in birthweight

\begin{tabular}{|c|c|c|c|c|}
\hline Variable & Model 1 & Model 2 & Model 3 & Model 4 \\
\hline$n$ & 3,972 & 3,972 & 3,972 & 3,404 \\
\hline Risk of $\mathrm{T} 2 \mathrm{D}^{\mathrm{a}}$ & $0.57(0.41,0.79)^{* * *}$ & $0.49(0.35,0.69)^{* * *}$ & $0.43(0.30,0.62)^{* * *}$ & $0.49(0.33,0.74)^{* * *}$ \\
\hline \multicolumn{5}{|l|}{ OGTT $^{\mathrm{b}}$} \\
\hline$n$ & 3,910 & 3,910 & 3,910 & 3,363 \\
\hline \multicolumn{5}{|l|}{ Glucose (\%) } \\
\hline $0 \mathrm{~min}$ & $-0.8(-1.5,-0.1)^{*}$ & $-1.1(-1.8,-0.4)^{* *}$ & $-1.2(-1.9,-0.5)^{* * *}$ & $-1.0(-1.7,-0.2)^{*}$ \\
\hline $30 \mathrm{~min}$ & $-2.2(-3.6,-0.9)^{* *}$ & $-2.7(-3.9,-1.4)^{* * *}$ & $-2.9(-4.2,-1.6)^{* * *}$ & $-2.3(-3.7,-0.9)^{* * *}$ \\
\hline $120 \mathrm{~min}$ & $-5.3(-7.1,-3.3)^{* * *}$ & $-6.0(-7.8,-4.2)^{* * *}$ & $-6.4(-8.2,-4.6)^{* * *}$ & $-5.6(-7.5,-3.6)^{* * *}$ \\
\hline AUC $0-120 \mathrm{~min}$ & $-2.9(-4.1,-1.7)^{* * *}$ & $-3.5(-4.6,-2.3)^{* * *}$ & $-3.8(-4.9,-2.6)^{* * *}$ & $-3.1(-4.4,-1.9)^{* * *}$ \\
\hline \multicolumn{5}{|l|}{ Insulin $(\%)$} \\
\hline $0 \mathrm{~min}$ & $-1.5(-5.4,2.6)$ & $-4.4(-7.3,-0.9)^{*}$ & $-4.7(-8.1,-1.2)^{* *}$ & $-5.4(-8.9,-1.7)^{* *}$ \\
\hline $30 \mathrm{~min}$ & $-1.7(-5.6,2.5)$ & $-3.8(-7.4,0.0)$ & $-2.9(-6.7,1.0)$ & $-2.6(-6.5,1.5)$ \\
\hline $120 \mathrm{~min}$ & $-16.6(-21.1,-11.9)^{* * *}$ & $-19.3(-23.3,-15.1)^{* * *}$ & $-19.4(-23.5,-15.1)^{* * *}$ & $-18.9(-23.2,-14.5)^{* * *}$ \\
\hline AUC $0-120 \mathrm{~min}$ & $-5.9(-9.4,-2.3)^{* *}$ & $-8.2(-11.3,-5.1)^{* * *}$ & $-7.9(-11.1,-4.7)^{* * *}$ & $-7.6(-10.9,-4.3)^{* * *}$ \\
\hline HOMA-IS (\%) & $2.3(-2.0,6.8)$ & $5.7(1.9,9.8)^{* *}$ & $6.1(2.1,10.3)^{* *}$ & $6.7(2.5,11.1)^{* *}$ \\
\hline Insulin sensitivity (\%) & $5.6(1.5,10.0)^{* *}$ & $9.1(5.5,12.8)^{* * *}$ & $9.3(5.6,13.2)^{* * *}$ & $9.1(5.2,13.0)^{* * *}$ \\
\hline Disposition index (\%) & $2.5(-0.4,5.4)$ & $3.7(0.9,6.5)^{* *}$ & $4.6(1.8,7.5)^{* *}$ & $4.1(1.1,7.2)^{* *}$ \\
\hline
\end{tabular}

Risk of T2D is provided by odds ratio (95\% CI). OGTT derived measures of glucose tolerance are provided as mean percentage change ( $95 \%$ CI) Model 1 was adjusted for age and sex; model 2 was further adjusted for current BMI; model 3 was additionally adjusted for maternal parity, and maternal and paternal history of diabetes; model 4 was additionally adjusted for socioeconomic status and lifestyle variables (physical activity, diet, alcohol consumption, smoking)

${ }^{\text {a }}$ Participants born at term with known or screen-detected type 2 diabetes

${ }^{\mathrm{b}}$ Participants with known type 2 diabetes at baseline visit were excluded from OGTT-derived measures of glucose tolerance ${ }^{*} p<0.05 ; * * p<0.01 ; * * * p<0.001$

HOMA-IS, HOMA of insulin sensitivity; T2D, type 2 diabetes

type 2 diabetes after adjusting for age, sex and BMI (Table 1). Adjustment for socioeconomic factors and lifestyle variables did not alter this observation. Supplementary analyses performed only on complete cases or using simple imputation of missing values for socioeconomic factors and lifestyle variables also did not change the observations (data not shown).

A $1 \mathrm{SD}\left(2.28 \mathrm{~kg} / \mathrm{m}^{3}\right)$ increase in ponderal index was associated with a $23 \%$ (OR $0.77 ; 95 \%$ CI $0.64-0.92$; $p<0.01)$ reduction in the risk of type 2 diabetes as compared with a $27 \%$ (OR $0.73 ; 95 \%$ CI $0.61-0.87$; $p<0.001)$ risk reduction related to an increase in birthweight of $1 \mathrm{SD}$ (451 g).

Birthweight was positively related to insulin sensitivity (Table 1). Despite an inverse relationship between birthweight and insulin release, birthweight showed a positive relationship with beta cell function, reflected by the disposition index (Table 1).

The participants born prematurely had a higher ponderal index at birth than SGA participants (Table 2). Despite this discrepancy, the two groups had similarly increased prevalence of type 2 diabetes and a similar degree of post-load hyperglycaemia, hyperinsulinaemia and insulin resistance. The OR for type 2 diabetes was 1.79 (95\% CI 1.17-2.74) for premature participants compared with participants born at term (SGA+appropriate for gestational age; $p=0.007)$. Birthweight $z$-scores, reflecting fetal growth rates, were not related to risk of type 2 diabetes or measures of glucose regulation among participants born prematurely (data not shown).

\section{Discussion}

Using a random cohort and a clinical assessment of glucose regulation and type 2 diabetes, this study demonstrates that low birthweight and prematurity are associated with increased risk of type 2 diabetes, supporting a recent registry-based study [5]. It also documents that low birthweight is associated with beta cell dysfunction and reduced insulin sensitivity, whereas prematurity is associated solely with attenuated insulin sensitivity.

As for the other end of the birthweight spectrum, elevated birthweight has been associated with type 2 diabetes in some 
Table 2 Adult size and glucose regulation in participants born prematurely, SGA or appropriate for gestational age

\begin{tabular}{|c|c|c|c|c|c|}
\hline \multirow[t]{2}{*}{ Characteristic } & \multicolumn{3}{|l|}{ Time of birth } & \multicolumn{2}{|c|}{$p$ value, premature } \\
\hline & Premature & Term SGA & Term AGA & vs SGA & vs $\mathrm{SGA}+\mathrm{AGA}$ \\
\hline$n$ (men) & 160 & 58 & 1,928 & & \\
\hline$n$ (women) & 283 & 88 & 2,127 & & \\
\hline Birthweight $(\mathrm{g})^{\mathrm{a}}$ & $2,555(403)$ & $2,556(152)$ & $3,522(420)$ & 0.99 & $<0.001$ \\
\hline Ponderal index $\left(\mathrm{kg} / \mathrm{m}^{3}\right)^{\mathrm{a}}$ & $22.8(2.3)$ & $22.0(1.9)$ & $24.9(2.2)$ & $<0.001$ & $<0.001$ \\
\hline Adult BMI $\left(\mathrm{kg} / \mathrm{m}^{2}\right)^{\mathrm{b}}$ & $25.8(4.7)$ & $25.2(4.1)$ & $26.2(4.5)$ & 0.17 & 0.41 \\
\hline Prevalence of T2D $(\%)^{\mathrm{c}}$ & 6.89 & 6.47 & 4.82 & 0.88 & 0.007 \\
\hline \multicolumn{6}{|l|}{ OGTT } \\
\hline \multicolumn{6}{|l|}{ Glucose $(\mathrm{mmol} / \mathrm{l})^{\mathrm{c}}$} \\
\hline $0 \mathrm{~min}$ & $5.54(1.01)$ & $5.48(0.67)$ & $5.51(0.74)$ & 0.76 & 0.011 \\
\hline $30 \mathrm{~min}$ & $8.63(1.82)$ & $8.61(1.86)$ & $8.62(1.82)$ & 0.49 & 0.035 \\
\hline $120 \min$ & $6.45(2.43)$ & $6.49(1.98)$ & $6.14(2.01)$ & 0.33 & 0.001 \\
\hline AUC 0-120 min & 888 (189) & $885(181)$ & $876(178)$ & 0.43 & 0.003 \\
\hline \multicolumn{6}{|l|}{ Insulin $(\mathrm{pmol} / 1)^{\mathrm{c}, \mathrm{d}}$} \\
\hline $0 \min$ & $33(23-49)$ & $31(23-47)$ & $35(23-49)$ & 0.94 & 0.12 \\
\hline $30 \mathrm{~min}$ & $242(180-374)$ & $239(170-371)$ & $234(165-339)$ & 0.65 & 0.004 \\
\hline $120 \mathrm{~min}$ & $193(110-292)$ & $160(106-282)$ & $149(91-240)$ & 0.057 & $<0.001$ \\
\hline AUC 0-120 min & $22,965(17,227-35,048)$ & $23,228(15,825-34,425)$ & $21,930(15,855-31,050)$ & 0.81 & $<0.001$ \\
\hline HOMA-IS ${ }^{\mathrm{c}, \mathrm{d}}$ & $0.89(0.56-1.27)$ & $0.94(0.60-1.28)$ & $0.87(0.57-1.28)$ & 0.97 & 0.053 \\
\hline Insulin sensitivity ${ }^{\mathrm{c}, \mathrm{d}}$ & $24.1(14.6-32.4)$ & $23.3(16.1-36.2)$ & $24.3(16.3-34.4)$ & 0.84 & $<0.001$ \\
\hline Disposition index ${ }^{\mathrm{c}, \mathrm{d}}$ & $629(471-815)$ & $675(473-823)$ & $623(483-801)$ & 0.77 & 0.80 \\
\hline
\end{tabular}

Unless otherwise indicated, data are means (SD) or medians (interquartile range); participants with known type 2 diabetes at baseline visit were excluded from OGTT-derived measures of glucose tolerance

${ }^{\mathrm{a}}$ Adjusted for sex; ${ }^{\mathrm{b}}$ adjusted for sex and age; ${ }^{\mathrm{c}}$ adjusted for sex, age and BMI; ${ }^{\mathrm{d}} \log _{\mathrm{e}}$ transformed prior to statistical analyses between groups AGA, appropriate for gestational age; T2D, type 2 diabetes

[12] but not all previous studies [1,2]. The lack of a significant relation between high birthweight and type 2 diabetes in this study may theoretically be explained by a low frequency of gestational diabetes among the mothers.

Ponderal index, a measure of thinness at birth, has previously been proposed to be a stronger predictor of type 2 diabetes than birthweight [2]. However, our finding of a quantitatively similar risk of type 2 diabetes for birthweight and ponderal index neither supports that notion nor the idea that ponderal index is a superior marker of an adverse intrauterine environment.

The robustness of our findings is illustrated by the consistency of the results across the four applied levels of adjustment. The finding that the associations between birthweight and risk of type 2 diabetes remained similar after adjusting for maternal parity and parental diabetes status (model 3), as well as socioeconomic and lifestyle factors (model 4), may be explained by the magnitude of findings for age and BMI, which are included in model 1 and 2 , respectively.

Originally, beta cell dysfunction was thought to represent a key link between low birthweight and the risk of type 2 diabetes [1]. However, subsequent studies reported low birthweight to be associated with reduced insulin sensitivity rather than decreased insulin secretion [13]. Our participants with low birthweight had elevated concentrations of post-load serum insulin, but when accounting for insulin sensitivity, their beta cell function was in fact reduced. The necessity of adjusting for insulin sensitivity may be particularly important in relatively young study populations like that of the Inter99 study, as hyperinsulinaemia and reduced insulin sensitivity precede pancreatic failure in absolute terms in the natural history of type 2 diabetes.

Despite less disproportionate fetal growth, reflected by a higher ponderal index at birth, participants born prematurely had a similar reduction of insulin sensitivity as well as a similarly elevated risk of type 2 diabetes compared with participants born SGA. Interestingly, no association between birthweight $z$ scores and type 2 diabetes or other measures of glucose regulation was observed among the participants born prematurely, and prematurity was not associated with reduced beta cell function. Altogether, our results suggest that the early life programming mechanisms 
that mediate the increased risk of type 2 diabetes may be different for low birthweight and prematurity.

The Inter99 study is based on an age- and sex-stratified random sample of the population. As in all populationbased studies, it faces selection bias towards more healthy individuals among participants compared with nonparticipants [6]. Moreover, the prevalence of type 2 diabetes was slightly lower among Inter99 participants with traced midwife records vs those without traced midwife records [8]. If anything, this selection bias towards inclusion of the healthiest segment of the Danish middleaged population may have led to underestimation of the reported effects of perinatal factors in the aetiology of type 2 diabetes.

In conclusion, we found both reduced insulin sensitivity and beta cell dysfunction to be mediators in the association between low birthweight and type 2 diabetes. In addition, prematurity was associated with attenuated insulin sensitivity and type 2 diabetes independently of fetal growth rate.

Acknowledgements The study was financially supported by research grants from the Öresund Diabetes Academy, the Danish Diabetes Association, the Danish Medical Research Council, the Danish Centre for Evaluation and Health Technology Assessment, Novo Nordisk, Glaxo SmithKline, Copenhagen County, the Danish Heart Foundation, the Danish Pharmaceutical Association, the Augustinus Foundation, the Ib Henriksen Foundation and the Becket Foundation. The Inter99 study was initiated by T. Jørgensen (principal investigator), K. Borch-Johnsen (co-principal investigator), H. Ibsen and $\mathrm{T}$. Thomsen. The Inter99 steering committee comprises T. Jørgensen, K. Borch-Johnsen and C. Pisinger. The authors thank the staff of Inter99 and all the participants.

Duality of interest K. Pilgaard, K. Færch, B. Carstensen, D. R. Witte and A. Vaag are employed at the Steno Diabetes Center, which is owned by Novo Nordisk A/S. O. Pedersen and T. Hansen are employed at Hagedorn Research Institute, which is owned by Novo Nordisk. All other authors declare that there is no duality of interest associated with this manuscript.

\section{References}

1. Hales CN, Barker DJ, Clark PM et al (1991) Fetal and infant growth and impaired glucose tolerance at age 64. BMJ, Br Med J 303:1019-1022

2. Lithell HO, McKeigue PM, Berglund L, Mohsen R, Lithell UB, Leon DA (1996) Relation of size at birth to non-insulin dependent diabetes and insulin concentrations in men aged 50-60 years. BMJ (Clinical research ed) 312:406-410

3. Phillips DI, Barker DJ, Hales CN, Hirst S, Osmond C (1994) Thinness at birth and insulin resistance in adult life. Diabetologia 37:150-154

4. Hofman PL, Regan F, Jackson WE et al (2004) Premature birth and later insulin resistance. N Engl J Med 351:2179-2186

5. Kaijser M, Bonamy AK, Akre O et al (2009) Perinatal risk factors for diabetes in later life. Diabetes 58:523-526

6. Jørgensen T, Borch-Johnsen K, Thomsen TF, Ibsen H, Glümer C, Pisinger C (2003) A randomized non-pharmacological intervention study for prevention of ischaemic heart disease: baseline results Inter99 (1). Eur J Cardiovasc Prev Rehab 10:377-386

7. Alberti KG, Zimmet PZ (1998) Definition, diagnosis and classification of diabetes mellitus and its complications. Part 1 : diagnosis and classification of diabetes mellitus provisional report of a WHO consultation. Diabet Med 15:539-553

8. Pilgaard K, Faerch P, Poulsen, C et al. (2010) Impact of size at birth and prematurity on adult anthropometry in 4744 middle-aged Danes - the Inter99 Study. Journal of Developmental Origins of Health and Disease doi:10.1017/S2040174410000413

9. Niklasson A, Ericson A, Fryer JG, Karlberg J, Lawrence C, Karlberg P (1991) An update of the Swedish reference standards for weight, length and head circumference at birth for given gestational age (1977-1981). Acta Paediatr Scand 80:756-762

10. Matthews DR, Hosker JP, Rudenski AS, Naylor BA, Treacher DF, Turner RC (1985) Homeostasis model assessment: insulin resistance and beta-cell function from fasting plasma glucose and insulin concentrations in man. Diabetologia 28:412-419

11. Matsuda M, DeFronzo RA (1999) Insulin sensitivity indices obtained from oral glucose tolerance testing: comparison with the euglycemic insulin clamp. Diab Care 22:1462-1470

12. McCance DR, Pettitt DJ, Hanson RL, Jacobsson LT, Knowler WC, Bennett PH (1994) Birth weight and non-insulin dependent diabetes: thrifty genotype, thrifty phenotype, or surviving small baby genotype? BMJ (Clinical research ed) 308:942-945

13. Cook JT, Levy JC, Page RC, Shaw JA, Hattersley AT, Turner RC (1993) Association of low birth weight with beta cell function in the adult first degree relatives of non-insulin dependent diabetic subjects. BMJ 306:302-306 